OPEN ACCESS

Edited by:

Thomas Fath

Macquarie University, Australia

Reviewed by:

Han-Rong Weng,

Mercer University, United States Lorenzo Di Cesare Mannelli,

University of Florence, Italy

*Correspondence:

Jun Chen

junchen@fmmu.edu.cn;

junchen_ibsp2015@yeah.net

Dong Huang

huangdong6619@vip.163.com

Specialty section: This article was submitted to Cellular Neuropathology, a section of the journal Frontiers in Cellular Neuroscience

Received: 12 April 2020 Accepted: 08 June 2020 Published: 30 June 2020

Citation:

Luo $X$, He T, Wang $Y$, Wang J-L, Yan $X-B$, Zhou $H-C$, Wang $R-R$,

Du R, Wang $X-L$, Chen $J$ and Huang D (2020) Ceftriaxone Relieves Trigeminal Neuropathic Pain Through Suppression of Spatiotemporal Synaptic Plasticity via Restoration of Glutamate Transporter 1 in the

Medullary Dorsal Horn. Front. Cell. Neurosci. 14:199. doi: 10.3389/fncel.2020.00199

\section{Ceftriaxone Relieves Trigeminal Neuropathic Pain Through Suppression of Spatiotemporal Synaptic Plasticity via Restoration of Glutamate Transporter 1 in the Medullary Dorsal Horn}

\author{
Xiao Luo ${ }^{1}$, Ting He ${ }^{2,3}$, Yan Wang ${ }^{2,3}$, Jiang-Lin Wang ${ }^{2,4}$, Xue-Bin Yan ${ }^{1}$, Hao-Cheng Zhou ${ }^{1}$, \\ Rui-Rui Wang ${ }^{2,3}$, Rui Du ${ }^{2}$, Xiao-Liang Wang ${ }^{2,3}$, Jun Chen ${ }^{2,3 *}$ and Dong Huang ${ }^{1 *}$ \\ ${ }^{1}$ Department of Pain Management, The Third Xiangya Hospital, Institute of Pain Medicine, Central South University, \\ Changsha, China, ${ }^{2}$ Institute for Biomedical Sciences of Pain, Tangdu Hospital, The Fourth Military Medical University, Xi'an, \\ China, ${ }^{3}$ Key Laboratory of Brain Stress and Behavior, People's Liberation Army, Xi'an, China, ${ }^{4}$ Department of Pain \\ Management, The Affiliated Hospital of Southwest Medical University, Luzhou, China
}

Using a rat model of trigeminal neuropathic pain (TNP) produced by chronic compression of the infraorbital nerve $(\mathrm{CCl}-\mathrm{ION})$, we investigated the analgesic effect and the underlying mechanisms of ceftriaxone (Cef), a $\beta$-lactam antibiotic, that is thought to be a potent stimulator of glutamate transporter 1 (GLT-1). First, repeated intraperitoneal (i.p.) injections of Cef $(200 \mathrm{mg} / \mathrm{kg})$ for 5 -days since Day 1 of CCI-ION could significantly relieve both mechanical and thermal pain hypersensitivity from day 10 after drug administration. Western blot and immunofluorescent results demonstrated that 5-days administration of Cef resulted in the restoration of GLT-1 expression to a level equivalent to the sham control which was dramatically lost under the TNP condition. Moreover, multi-electrode $(8 \times 8)$ array recordings of network field excitatory postsynaptic potentials (fEPSPs) were performed on the acutely dissociated medullary dorsal horn slice evoked by electrical stimulation of the trigeminal spinal tract. The results showed that the increased number of fEPSPs, induction rate, and maintenance of long-term potentiation caused by CCl-ION were significantly suppressed by 5-days administration of Cef. Taken together, the results indicate that Cef can relieve TNP through suppression of spatiotemporal synaptic plasticity via GLT-1 restoration in the medullary dorsal horn of the trigeminal nerve.

Keywords: trigeminal neuropathic pain, glutamate transporter 1, ceftriaxone, synaptic plasticity, long-term potentiation, medullary dorsal horn

\section{INTRODUCTION}

Trigeminal neuralgia (TN) is one of the most intense orofacial pain affecting one or more branches of the 5th cranial nerve (Finnerup et al., 2010; Scholz et al., 2019). The causes and pathology of trigeminal neuralgia are not well understood and the treatment is still a major challenge. Carbamazepine is the first-line drug recommended by the American Academy of Neurology 
and the European Federation of Neurological Societies which can effectively alleviate TN, however, the side-effects become apparent with the emergence of drug resistance (Finnerup et al., 2010; Scholz et al., 2019). A substantial proportion of patients have to abandon the drug and choose surgical treatment. The treatment will become extremely troublesome for the patients with recurrent symptoms after surgery, while the recurrent rate ranges from $20 \%$ to $50 \% 5$ years after surgery (Tronnier et al., 2001; Cheng et al., 2014). So, more effective and novel drugs are required to replace the traditional ones.

Glutamate transporter 1 (GLT-1) is a sodium-dependent high-affinity glutamate transporter which is abundantly localized in astrocytes and axon-terminals in some parts of the mature brain (Rothstein et al., 1994; Chen et al., 2002, 2004; Furness et al., 2008; Rose et al., 2009). In the spinal cord, GLT-1 is also largely found in astrocytes and growing axonal fibers especially in the gray matter (Rothstein et al., 1994; Danbolt, 2001; Yan et al., 2014). Glutamate is the major excitatory neurotransmitter participating in nociceptive processing and synaptic plasticity during which trafficking of ionotropic glutamate receptors may occur (Malinow and Malenka, 2002; Larsson and Broman, 2011; Liu and Salter, 2010). Both release and uptake abnormalities of glutamate were observed in neuropathic pain (Inquimbert et al., 2012; Di Cesare Mannelli et al., 2015). GLT-1 is crucial for the modulation of the dynamic balance of neurotransmitters in the synaptic cleft, together with glutamate/aspartate transporter (GLAST) to accomplish more than $95 \%$ of glutamate uptaking (Danbolt, 2001; Kanai et al., 2013). In case of GLT-1 downregulation, the extracellular glutamate concentrations would be increased, resulting in excitotoxic neuronal damage that can be happening in the process of amyotrophic lateral sclerosis (Rothstein et al., 1995), spinal trauma (Rao et al., 1998; Lepore et al., 2011) and neuropathy (Jacob et al., 2007; Hazell et al., 2010).

Previous studies have demonstrated that the abnormal expression of GLT-1 was associated with the development of neuropathic pain in various animal models, such as chronic constriction injury of the sciatic nerve (SN-CCI; Hu et al., 2010; Suzuki et al., 2012), ligation of spinal nerves (SNL; Hobo et al., 2011) and spared nerve injury (SNI) of the sciatic nerve (Inquimbert et al., 2012). A transient up-regulation of GLT-1 was observed in some pain models at the initial phase (Yamada et al., 1998; Cavaliere et al., 2007), which was thought to be a compensatory effect in response to the increasing glutamate release. However, the expression of GLT-1 was stably down-regulated at the later phase of the peripheral nerve injury (Weng et al., 2014; Yan et al., 2014). The down-regulation of GLT-1 contributes to an increase in glutamate concentration at the synaptic cleft, which may result in the activation of distant spinal neurons and astrocytic cells (Nie and Weng, 2010; Nie et al., 2010). Excessive extracellular glutamate accumulation has been shown to trigger intracellular $\mathrm{Ca}^{2+}$ release in astrocytic cells (Rojas et al., 2007; Yoshizumi et al., 2012), that may magnify the primary afferents. Based on the findings above, GLT-1 is regarded as a promising therapeutic target in neuropathic pain (Hu et al., 2010; Ramos et al., 2010; Nicholson et al., 2014; Chelini et al., 2017; Butler, 2018).
Growing evidence indicates that astrocytes regulate synaptic transmission and plasticity through modulation of excitatory-inhibitory balance, especially the increased efficacy of glutamatergic neurotransmission (Bonansco and Fuenzalida, 2016). Under the neuropathic conditions, such as epilepsy, glutamate-mediated gliotransmission was considered to be a putative signal of increased neuronal excitability (Tang and Lee, 2001). By uptaking glutamate in the synaptic cleft, glutamate transporter is likely to maintain excitatory-inhibitory balance and prevent aberrant signals which may trigger a cascade of plasticity events. The previous studies have proved that GLT-1 plays a critical role in LTP induction and maintenance of long-term changes of synaptic efficacy (Katagiri et al., 2001; Levenson et al., 2002). However, the precise regulation of GLT-1 in astrocyte-neuron communication and synaptic plasticity remains to be explored. It is thus hypothesized that nerve injury-induced GLT-1 changes may alter the spatiotemporal characteristics and coding of nociceptive signals.

The $\beta$-lactam antibiotic ceftriaxone (Cef), which can increase the expression of GLT-1 in vivo and in vitro (Rothstein et al., 2005). In previous studies, Cef was shown to reverse the down-regulation of GLT-1 and to elevate the glutamate uptake in chronic pain models, subsequently resulting in an analgesic effect (Hu et al., 2010; Nicholson et al., 2014; Butler, 2018). The compounds of the Cef are expected to be a class of promising analgesic drugs that can be in combination with opioid analgesics to reduce tolerance (Rawls et al., 2010). Pharmacological inhibition of GLT-1 by dihydrokainate can reverse the analgesic effect of Cef in diabetic rats (Gunduz et al., 2011). Moreover, Cef can exert its therapeutic effect by reducing glial activation in neuropathic pain (Ramos et al., 2010; Nicholson et al., 2014). However, the exact therapeutic effects of Cef and its modulation of the glutamate transporter system in the TN is still not clear. Therefore, we designed experiments and looked at the following aspects of Cef: (1) anti-nociceptive effects of long-term administration of Cef in a rat model of $\mathrm{TN}$; (2) effects of long-term administration of Cef on the expression level of GLT-1 in the trigeminal nucleus of the spinal tract caudalis (Sp5C), which is known as the medullary dorsal horn receiving primary nociceptive afferents from the semilunar ganglion sensory cells of the trigeminal nerve; (3) effects of long-term administration of Cef on the spatiotemporal network responses of excitatory postsynaptic field potentials (fEPSPs) evoked by electrical stimulation of the Sp5 recorded on medullary dorsal horn slice using multi-electrode array (MEA, $8 \times 8$ ) recording system.

\section{MATERIALS AND METHODS}

\section{Animals and Surgery}

Experiments were carried out on male albino Sprague-Dawley rats (3-4 weeks old, 80-120 g) provided by the Laboratory Animal Center of Fourth Military Medical University (FMMU). Animals were housed in a group of five per cage under standard laboratory conditions $(12: 12 \mathrm{~h}$ day/night cycle, with a temperature of $22-26^{\circ} \mathrm{C}$ and air humidity of 
55-60\%), with access to food and water ad libitum. All the experiments were approved by the Institutional Animal Care and Use Committee at the FMMU and were as per the recommendations of the ARRIVE guidelines, the U.K. Animals (Scientific Procedures) Act 1986 and associated guidelines, the EU Directive 2010/63/EU for animal experiments, the National Institutes of Health Guide for Care and Use of Laboratory Animals (Publication No. 85-23, revised 1996). The ethical guidelines for investigations of experimental pain in conscious animals of the International Association for the Study of Pain were also critically followed. Efforts have been made to minimize the number of animals used and their suffering.

To prepare a model of trigeminal neuropathic pain (TNP), unilateral ligation of the infraorbital nerve (ION) was performed following the protocols described by Imamura (Imamura et al., 1997). The rats were anesthetized with pentobarbital sodium (50 mg/kg, i.p.) and an incision was made intraorally along the gingivobuccal margin to expose the ION (Figure 1A). A double ligature was tied loosely around the ION with 5-0 chromic guts (2 mm apart). After ligation, the incision was sutured using silk. In sham-operated rats, the ION was isolated using the same procedure except that the nerve was not ligated.

\section{Behavioral Tests}

The behavioral assays were performed before and post-surgery from day 1 to day 14 (Figure 1A). The rats were individually placed in a plastic cage $1 \mathrm{~h}$ before the test to adapt to the environment. A set of von Frey filaments were used to measure the mechanical sensitivity of the facial whisker pad as previously described, according to the up-and-down method with the cut-off intensity of $15 \mathrm{~g}$ (Vos et al., 1994). The thermal sensitivity of the facial whisker pad was evaluated using the published method (Imamura et al., 1997). A special box with a hole at the front which allows the rat's snout to poke through was prepared, besides the hole, the other part of the front-end was covered with a white paper that occluded the rat's vision when its snout protruded through the hole. The thermal stimulus was given to the centra of the whisker pad that can raise the skin temperature to $45-50^{\circ} \mathrm{C}$ in $10 \mathrm{~s}$. The cut-off time was set at $20 \mathrm{~s}$ to prevent tissue damage. Thermal withdrawal latency was measured three times for each rat at intervals of $2 \mathrm{~min}$.

\section{Slice Preparation}

Rats were anesthetized with pentobarbital sodium $(50 \mathrm{mg} / \mathrm{kg}$, i.p.) and then perfused with oxygenated N-methyl-D-glucamine

A

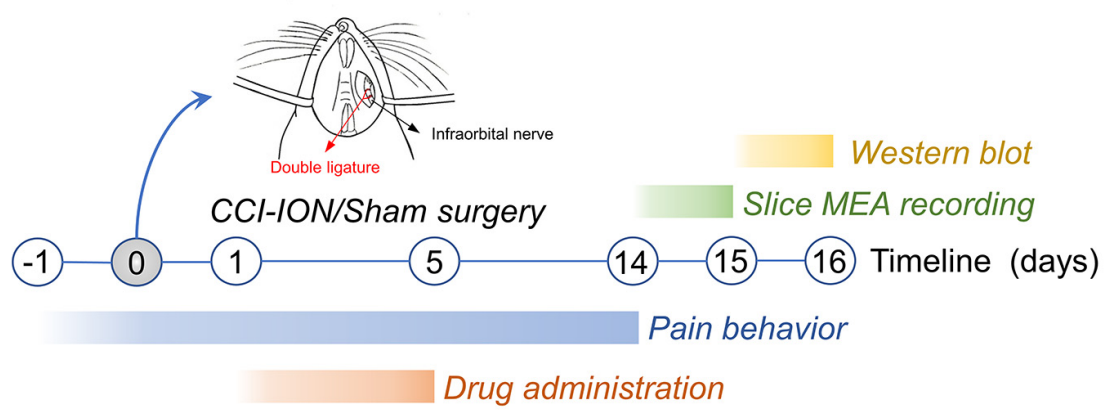

B

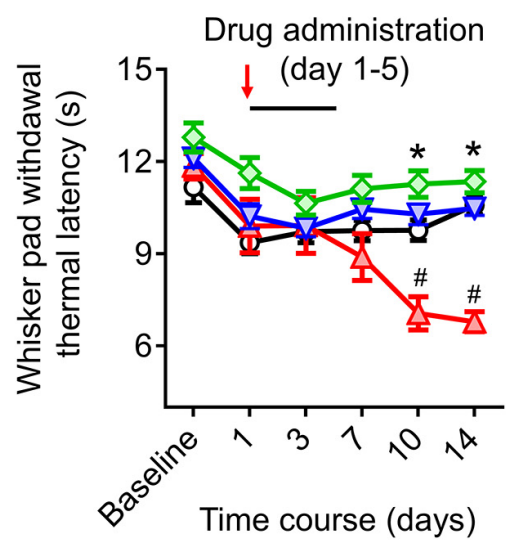

C

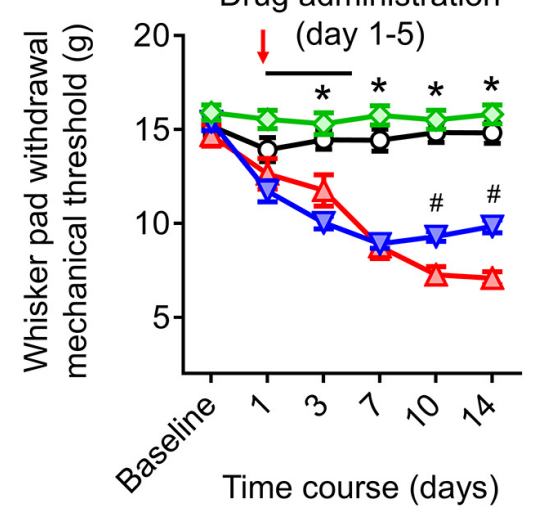

O Sham+saline (18)

$\triangle \mathrm{CCl}+$ saline (18)

$\nabla \mathrm{CCl}+\mathrm{Cef}(18)$

$\checkmark$ Sham+Cef (18)

FIGURE 1 | Timeline of the experimental design and procedures, as well as the effects of repeated systemic administration of ceftriaxone (Cef) on CCI-ION-induced pain hypersensitivity. (A) Timeline of the experimental design and procedures (for details see "Materials and Methods" section in the text). (B,C) Changes in whisker pad withdrawal thermal latency and mechanical threshold in rats with Sham + saline $(n=18), \mathrm{CCl}$-saline $(n=18), \mathrm{CCl}$-Cef $(n=18)$ and Sham $+\mathrm{Cef}(n=18)$. Arrows indicate the start of the drug administration. Data are presented as mean $\pm \mathrm{SEM} .{ }^{*} p<0.05, \mathrm{CCl}+\mathrm{saline} v \mathrm{v}$. Sham + saline; ${ }^{\#} p<0.05, \mathrm{CCl}+\mathrm{Cef}$ vs. CCI + saline. $\mathrm{CCI}-\mathrm{ION}$, chronic constriction injury of infraorbital nerve; MEA, multi-electrode array. 
(NMDG) artificial cerebrospinal fluid (NMDG ACSF) containing $92 \mathrm{mM}$ NMDG, $2.5 \mathrm{mM} \mathrm{KCl}, 1.25 \mathrm{mM} \mathrm{NaH}_{2} \mathrm{PO}_{4}$, $30 \mathrm{mM} \mathrm{NaHCO} 3,20 \mathrm{mM}$ HEPES, $25 \mathrm{mM}$ glucose, $2 \mathrm{mM}$ thiourea, $5 \mathrm{mM}$ Na-ascorbate, $3 \mathrm{mM}$ Na-pyruvate, $0.5 \mathrm{mM}$ $\mathrm{CaCl}_{2}$, and $10 \mathrm{mM} \mathrm{MgCl}_{2} \cdot 6 \mathrm{H}_{2} \mathrm{O}(\mathrm{pH} 7.3-7.4)$ according to Ting's protocol (Ting et al., 2014), the medullary and upper cervical spinal tissue was quickly isolated and submerged into pre-oxygenated NMDG ACSF. The tissue was fixed in an agar stage with cyanoacrylate glue to stick on the plate and then cut into $400 \mu \mathrm{m}$ coronal slices with a Vibratome (Dosaka, DTK-1000, Japan). Slices were transferred to a chamber continuously perfused with oxygenated HEPES holding ACSF containing $92 \mathrm{mM} \mathrm{NaCl}, 2.5 \mathrm{mM} \mathrm{KCl}, 1.25 \mathrm{mM} \mathrm{NaH}_{2} \mathrm{PO}_{4}$, $30 \mathrm{mM} \mathrm{NaHCO} 3,20 \mathrm{mM}$ HEPES, $25 \mathrm{mM}$ glucose, $2 \mathrm{mM}$ thiourea, $5 \mathrm{mM} \mathrm{Na}$-ascorbate, $3 \mathrm{mM}$ Na-pyruvate, $2 \mathrm{mM} \mathrm{CaCl}_{2}$, and $2 \mathrm{mM} \mathrm{MgCl} 2 \cdot 6 \mathrm{H}_{2} \mathrm{O}$. Before MEA recording, slices were recovered for at least $2 \mathrm{~h}$ at room temperature.

\section{Electrophysiological Recordings}

The electrophysiological recordings were performed on days 14-15 after surgery (Figure 1A). A $64(8 \times 8)$ array multielectrode dish system (MED-64, Alpha-Med Scientific, Japan) was used to record fEPSPs on a medullary slice containing the Sp5C (Figure 4A). After recovery in the HEPES holding ACSF for $2 \mathrm{~h}$, the slice was transferred to the MED-64 probe (P515A, $50 \times 50 \mu \mathrm{m}, 150 \mu \mathrm{m}$ interval) to cover the medullary dorsal horn and a nylon mesh anchor was covered to the slice to ensure stability. The slice was continuously perfused with oxygenated recording ACSF containing $119 \mathrm{mM} \mathrm{NaCl}$, $2.5 \mathrm{mM} \mathrm{KCl}, 1.25 \mathrm{mM} \mathrm{NaH}_{2} \mathrm{PO}_{4}, 24 \mathrm{mM} \mathrm{NaHCO}_{3}, 12.5 \mathrm{mM}$ glucose, $2 \mathrm{mM} \mathrm{CaCl}$, and $2 \mathrm{mM} \mathrm{MgCl}_{2} \cdot 6 \mathrm{H}_{2} \mathrm{O}$ at a flow rate of $2 \mathrm{ml} / \mathrm{min}$ with a peristaltic pump (PERI-STARTM, PI, USA) at room temperature. After 10-15 min adaption, at least one channel was arranged over the Sp5 allowing electrical stimulation with the aid of visual localization through a chargecoupled device camera (DP70, Olympus, Japan). A biphasic square-wave pulse $(0.1 \mathrm{~Hz}, 0.2 \mathrm{~ms}, 10-199 \mu \mathrm{A})$ generated by the data acquisition software (Conductor 3.0, Panasonic Alpha-Med Sciences, Japan) was applied to the electrical stimulus site, the evoked fEPSPs were amplified by the 64-channel amplifier and digitized at $20 \mathrm{kHz}$ sampling rate. The input-output (I-O) curve was acquired by measurements of the fEPSPs amplitude and slope in response to the gradient stimulation from 20 to $199 \mu \mathrm{A}$ with a $20 \mu \mathrm{A}$ stepwise increase. Higher intensities $(>199 \mu \mathrm{A})$ were not applied due to the limitation of the stimulus generator. The stimulus intensity for the test was then adjusted to enable produce $40-60 \%$ of maximal response based on the I-O curve. As for long-term potentiation (LTP) induction, the high-frequency stimulation (HFS, 10 bursts/s, $4 \times 0.2 \mathrm{~ms}$ pulses with $60 \mu \mathrm{A}$ at $100 \mathrm{~Hz}$ for each burst) protocol was used as described before (Heusler et al., 2000; Hjornevik et al., 2008; Zhao et al., 2009). The test stimulus was repeatedly delivered once every $10 \mathrm{~min}$ for $90 \mathrm{~min}$ and the responses were measured as an average of five individual traces. In another set of experiments, CNQX $(10 \mu \mathrm{M})$ and (TTX $0.5 \mu \mathrm{M}$ ) were perfused to the slices at a rate of $2 \mathrm{ml} / \mathrm{min}$ to identify whether the network field potentials are ionic glutamate receptor-mediated fEPSPs. After that, the slices were washed by fresh ACSF and tested until the responses tended to be stable. The details of the MED-64 system usage can be referred to our previous publications (Zhao et al., 2009; Wang et al., 2010; Yu et al., 2017).

For the quantification of the I-O relationship, the amplitude and slope of fEPSPs were analyzed by the MED-64 conductor. The total number of effective fEPSPs (>20\% baseline) was counted by an experimenter unaware of the experimental design and averaged across slices for each group. For induction of LTP, a $20 \%$ increase in the amplitudes of fEPSPs over the baseline that could be maintained for more than 30 min was set as successful induction (Figure 6B). The amplitudes of fEPSP were normalized and expressed as a percentage to the baseline value.

\section{Western Blot}

Medullary slices of each group were quickly removed and washed by PBS after MED-64 recording (Figure 1A), and then the tissue was homogenized in RIPA lysis buffer for $10 \mathrm{~min}$ in an ice bath. The lysates were centrifuged for $10 \mathrm{~min}$ at $12,000 \mathrm{rpm}$ at $4^{\circ} \mathrm{C}$. A BCA protein assay kit was used to measure the protein concentration. The samples (50 $\mu$ g protein) were placed on the SDS-PAGE gel, run at $80 \mathrm{~V}$ for $30 \mathrm{~min}$ and then $120 \mathrm{~V}$ for $1 \mathrm{~h}$, and then transferred to nitrocellulose membrane (Bio-Rad). After block with 5\% nonfat milk in PBS for $1 \mathrm{~h}$, the membrane was incubated overnight at $4^{\circ} \mathrm{C}$ with primary rabbit anti-GLT- 1 antibody (1:1,000, Abcam, UK) and mouse anti- $\beta$ actin (1:2,000, Abcam). After three times of washing (10 min each) in PBST, the membranes were incubated with secondary HRP-conjugated goat-anti-rabbit antibody (1:2,000, Bio-Rad) for $2 \mathrm{~h}$ at room temperature. The membranes were treated with enhanced chemiluminescence solution (Alpha Innotech Corp., USA) and the signals were detected by FluorChem FC2 (Alpha Innotech Corp). The density of each band was measured using a computer-assisted imaging analysis system (Bio-Rad, CA, USA) and normalized to $\beta$-actin intensity.

\section{Immunofluorescence}

Tissues were obtained 10 days after CCI-ION surgery. After anesthetized with pentobarbital sodium (50 $\mathrm{mg} / \mathrm{kg}$, i.p.), the rats were transcardially perfused with PBS, followed by $4 \%$ paraformaldehyde in $0.1 \mathrm{M} \mathrm{PB}$ solution. The medulla oblongata was removed and placed in $4 \%$ paraformaldehyde overnight at $4^{\circ} \mathrm{C}$, and then transferred to $30 \%$ sucrose in PBS for cryoprotection. The tissues were cut into coronal sections (25 $\mu \mathrm{m})$ on CM1900 freezing microtome (Leica, Germany). The sections were rinsed in PBS and blocked with $10 \%$ goat serum in PBS for $2 \mathrm{~h}$, and then incubated in primary antibodies overnight at $4^{\circ} \mathrm{C}$. Washed by PBS for three times $(10 \mathrm{~min}$ each), the sections were incubated with secondary antibodies for $2 \mathrm{~h}$ in a dark environment at room temperature. The primary antibodies used in the present study were rabbit anti-GLT-1 (1:200, Abcam, UK) and mouse anti-glia fibrillary acidic protein (GFAP; 1:200, Sigma-Aldrich, St. Louis, MO, USA). The secondary antibodies were Cy3 (552 nm) anti-rabbit (1:100, Sigma-Aldrich, St. Louis, MO, USA) and fluorescein isothiocyanate isomer (FITC; $494 \mathrm{~nm}$ ) anti-mouse. After that, DAPI (1:2,000, Sigma-Aldrich, St. Louis, 
MO, USA) was added to the sections for $10 \mathrm{~min}$. The sections were washed by PBS and mounted on slides. A laser scan confocal fluorescent microscope (Olympus FV1000, Japan) was used to acquire the images and the fluorescence intensity was measured by an image processing program ImageJ (National Institutes of Health, Bethesda, MD, USA).

\section{Drugs}

The $\beta$-lactam antibiotic ceftriaxone sodium (Cef) was purchased from Roche Corp (Switzerland) and dissolved in saline solution $(0.9 \% \mathrm{NaCl})$ at $0.2 \mathrm{~g} / \mathrm{mL}$. The Cef solution was administered by intraperitoneal (i.p.) injection $(200 \mathrm{mg} / \mathrm{kg})$. Cef administration started from day 1 after CCI-ION surgery and continued for five consecutive days (Figure 1A).

\section{Statistics}

Data were presented as mean \pm SEM. One way ANOVA with LSD post hoc corrections and independent-sample $t$-test (twotailed) was used for parametric data, while Kruskal-Wallis one way ANOVA test with Bonferroni post hoc corrections and Mann-Whitney $U$-test were used for non-parametric data according to the results of normality Shapiro-Wilk test and equal variance Levene test, and Pearson Chi-square tests with Fisher exact tests were used for fourfold table data (for details see Supplementary Table S1). All statistical analyses were performed used SPSS 25.0 software. $P<0.05$ was considered to be statistically significant. The artwork was performed by GraphPad Prism 6.01 (GraphPad Software Inc., San Diego, CA, USA).

\section{RESULTS}

\section{Anti-nociceptive Effects of Repeated Systemic Administration of Cef on the CCI-ION-Induced Hyperalgesia}

Compared with the sham group, rats with CCI-ION developed both thermal and mechanical pain hypersensitivity since 10 days
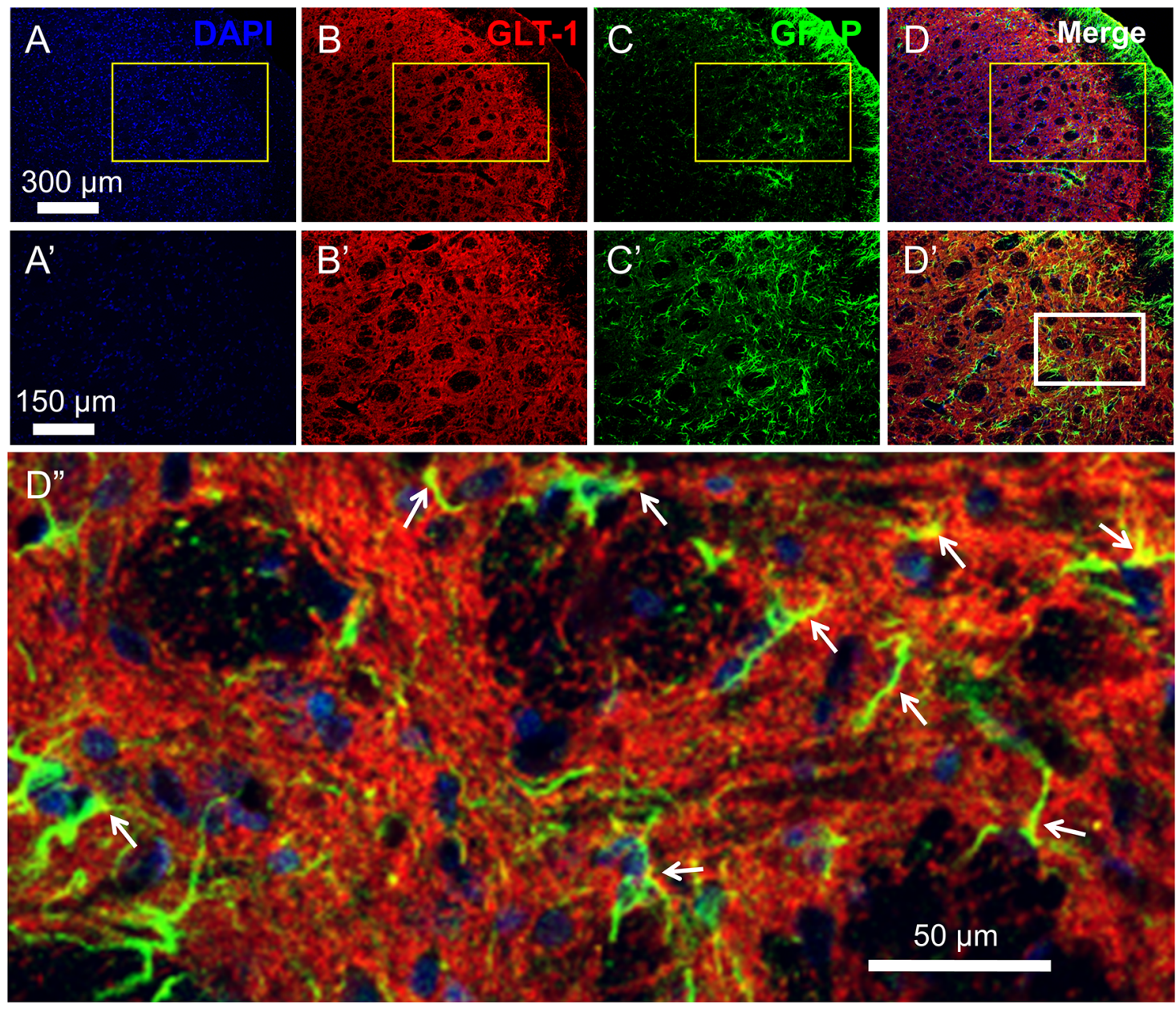

FIGURE 2 | Distribution and co-localization of glutamate transporter 1 (GLT-1) and glia fibrillary acidic protein (GFAP)-labeled profiles in the medullary dorsal horn. Panels (A-D) show triple labeling of DAPI (blue), GLT-1 (red) and GFAP (green). Panesl (A'-D') show insets from panels (A-D). Panel (D") shows inset from panel (D'). Scale $=300 \mu \mathrm{m}$ for (A-D); $150 \mu \mathrm{m}$ for (A'-D') and $50 \mu \mathrm{m}$ for (D”). Arrows indicate co-localization of GLT-1 and GFAP (yellow) in astrocytic profiles. 
after surgery (Figures 1B,C, ${ }^{*} p<0.001$ and ${ }^{*} p<0.001$, CCI + saline vs. Sham + saline, for details of statistical analysis see Supplementary Table S1). However, the CCI-IONinduced thermal and mechanical pain hypersensitivity were both suppressed by 5-days i.p. administrations of Cef relative to vehicle control (Figures 1B,C, ${ }^{*} p<0.001$ and ${ }^{\#} p=0.001$ in day 10, CCI + Cef vs. CCI + saline, for details of statistical analysis see Supplementary Table S1). The same treatment of Cef did not have any effects on the basal mechanical and thermal sensitivity (Figures 1B,C, see Supplementary Table S1). Time course observations revealed that the thermal pain hypersensitivity was more sensitive to the Cef treatment than mechanical pain hypersensitivity (Figures 1B,C).

\section{Reversal Effects of Repeated Systemic Administration of Cef on CCl-ION-Induced Loss of GLT-1 Expression in the Spinal Dorsal Horn}

As previously reported, double immunofluorescent labeling showed co-localization of GLT-1 and GFAP, a specific marker of astrocytes, in the medullary dorsal horn of rats (Figure 2). Compared with sham control, CCI-ION resulted in significant loss of GLT-1 protein expression in the medullary dorsal horn when assayed on day 15 after surgery (Figures $3 \mathbf{A , B}$, ${ }^{*} p=0.009$, CCI + saline vs. Sham + saline, see Supplementary
Table S1). However, the CCI-ION-induced decrease in GLT-1 expression was fully reversed by 5-days i.p. administrations of Cef relative to vehicle control (Figures 3A-D, ${ }^{*} p<0.001$ for immunofluorescent labeling and ${ }^{\#} p=0.009$ for Western blot, CCI + Cef vs. CCI + saline, for details of statistical analysis see Supplementary Table S1). Similar to the behavioral test, the same treatment of the Cef did not have any effects on the level of GLT-1 expression in the sham group of rats (Figures 3A,B, see Supplementary Table S1).

\section{Reversal Effects of Repeated Systemic Administration of Cef on CCI-ION-Induced Enhanced Network fEPSPs Response and LTP Induction and Maintenance}

Figure 4A showed an arrangement of MED-64 recording dish with 64 channels $(8 \times 8$ array) over the medullary dorsal horn slice wherein one channel (see red dot) was remained for electrical stimulation of the Sp5, while the other channels were used for simultaneous recordings of network fEPSPs in the medullary dorsal horn (see lower panel of Figure 4A for the typical waveform of an fEPSP recorded from 51\# channel, arrows for $1 \#-8 \#$ and $56 \#-64 \#$ on the upper panel indicate the order of electrode array). The $\mathrm{Sp} 5$-activated local network field potentials could be completely blocked by TTX (Figure 4B) and partially blocked by CNQX (Figure 4C), suggesting mediation of network
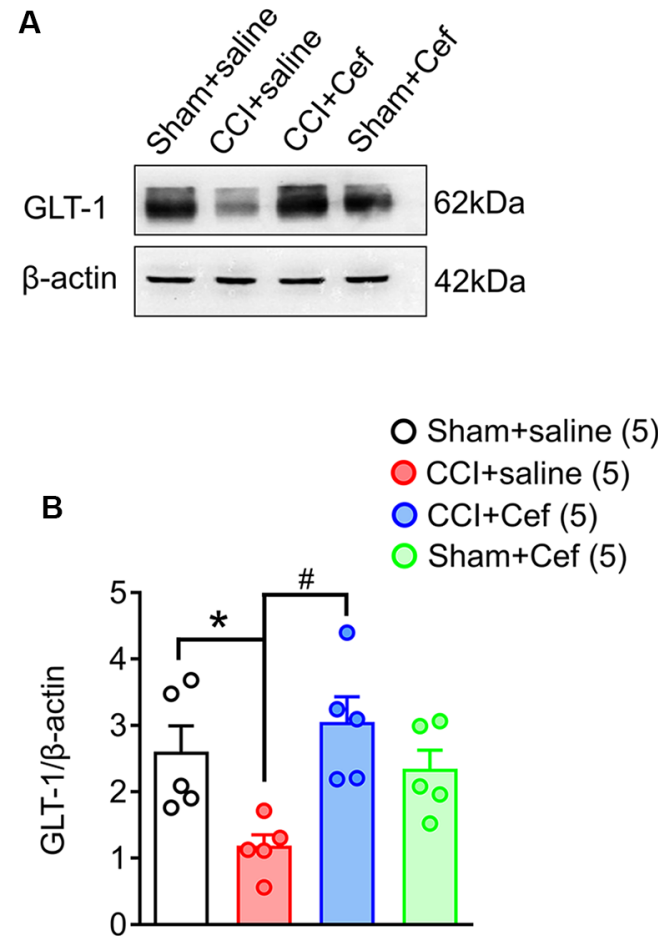

C
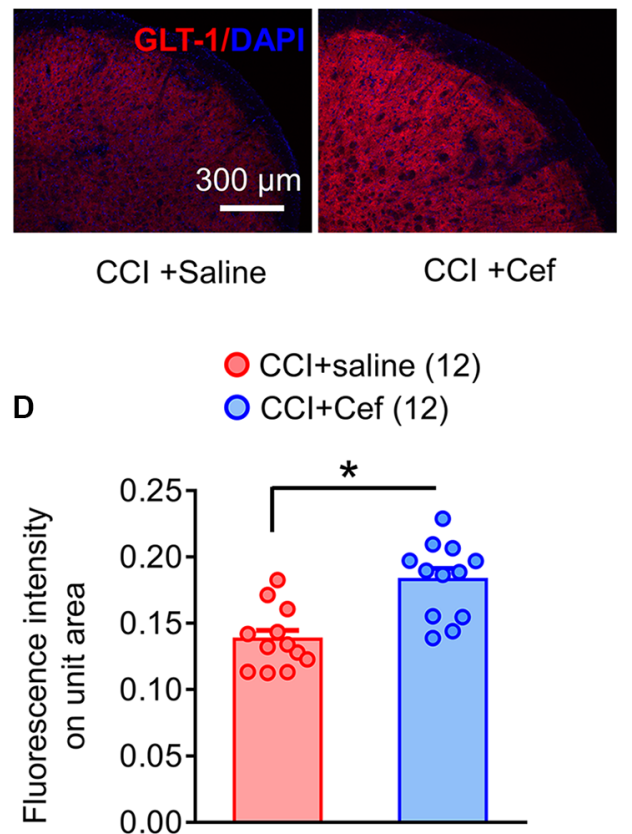

FIGURE 3 | Reversal effects of repeated systemic administration of ceftriaxone (Cef) on CCI-ION-induced loss of GLT-1 protein in the medullary dorsal horn. Panels (A,B) show expression of GLT-1 protein in the ipsilateral medullary dorsal horn of rats with Sham + saline $(n=5)$, CCl-saline $(n=5)$, CCl-Cef $(n=5)$ and Sham + Cef $(n=5)$. Data are presented as mean \pm SEM. ${ }^{*} p<0.05, \mathrm{CCl}+$ saline vs. Sham + saline; ${ }^{*} p<0.05, \mathrm{CCl}+\mathrm{Cef}$ vs. CCl + saline. (C,D) Immunofluorescent staining of GLT-1 (red) and DAPI (blue) in the ipsilateral medullary dorsal horn of CCl + saline $(n=12)$ and CCl + Cef $(n=12)$ groups. Data are shown as mean \pm SEM. ${ }^{*} p<0.05, \mathrm{CCl}+$ Cef vs. CCl + saline. GLT-1, glutamate transporter 1 . 
fEPSPs by both action potentials produced by activation of voltage-gated sodium channels (VGSCs) and ionic non-NMDA and probably NMDA glutamate receptors (Zhao et al., 2009; Wang et al., 2010; Yu et al., 2017).

Following 14-15 days after the CCI-ION, the spatial number of the Sp5-evoked fEPSPs was significantly increased when tested by a higher intensity of electrical stimulation $(140-200 \mu \mathrm{A})$ relative to sham control (Figures 5A,B,D, ${ }^{*} p=0.029 \mathrm{CCI}+$ saline vs. Sham + saline, see Supplementary Table S1). The I-V curve was leftward shifted in a group of CCI-ION relative to the sham control as well (Figures $\mathbf{5 A , B , E},{ }^{*} p=0.004$ CCI + saline vs. Sham + saline, see Supplementary Table S1). However, repeated i.p. treatment with Cef reversed both the CCI-IONexpanded spatial network fEPSPs response (Figures 5B-D, ${ }^{\#} p=0.027$ CCI + Cef vs. CCI + saline, see Supplementary Table S1) and CCI-ION-produced leftward-shift of I-V curve (Figures $5 \mathrm{~B}, \mathrm{C}, \mathrm{E},{ }^{*} p=0.027 \mathrm{CCI}+\mathrm{Cef}$ vs. CCI + saline, see Supplementary Table S1), compared to the vehicle control.

As for the effects of Cef-treatment on the induction and maintenance of LTP, conditioning stimulation was applied through electrical stimulation electrode (10 bursts/s, $4 \times 0.2 \mathrm{~ms}$ pulses at $100 \mathrm{~Hz}, 60 \mu \mathrm{A}$ for each burst). As shown in Figure 6A, the induction rate of LTP was also dramatically increased in the CCI-ION group by about $77 \%$ relative to $15.4 \%$ in the sham group $(p=0.005$, CCI + saline vs. Sham + Saline, see Supplementary Table S1). However, the increased induction rate of LTP was distinctly reversed by repeated systemic treatment of Cef (Figure 6A, $P=0.030$, CCI + Cef vs. CCI + Saline: $33.33 \%$ vs. $76.92 \%$, see Supplementary Table S1). Cef-treatment could not change the induction rate in sham control (Figure 6A, $P=0.221$, Sham + Cef vs. Sham + Saline: $40.00 \%$ vs. $15.38 \%$, see Supplementary Table S1). The CCI-ION resulted in enhancement of LTP in terms of both amplitude and time course relative to the sham control (Figure 6B, $P=0.001$, CCI + Saline vs. Sham + Saline, see Supplementary Table S1), while repeated systemic treatment of Cef produced a reversal effect on such enhancement compared to the vehicle control (Figure 6B, $P=0.024$, CCI + Cef vs. CCI + Saline, see Supplementary Table S1).

\section{DISCUSSION}

The major gains of the current study are as follows: (1) repeated systemic administration of Cef could mitigate both orofacial thermal and mechanical pain hypersensitivity caused by CCI-ION in rats, while the same treatment did not have any
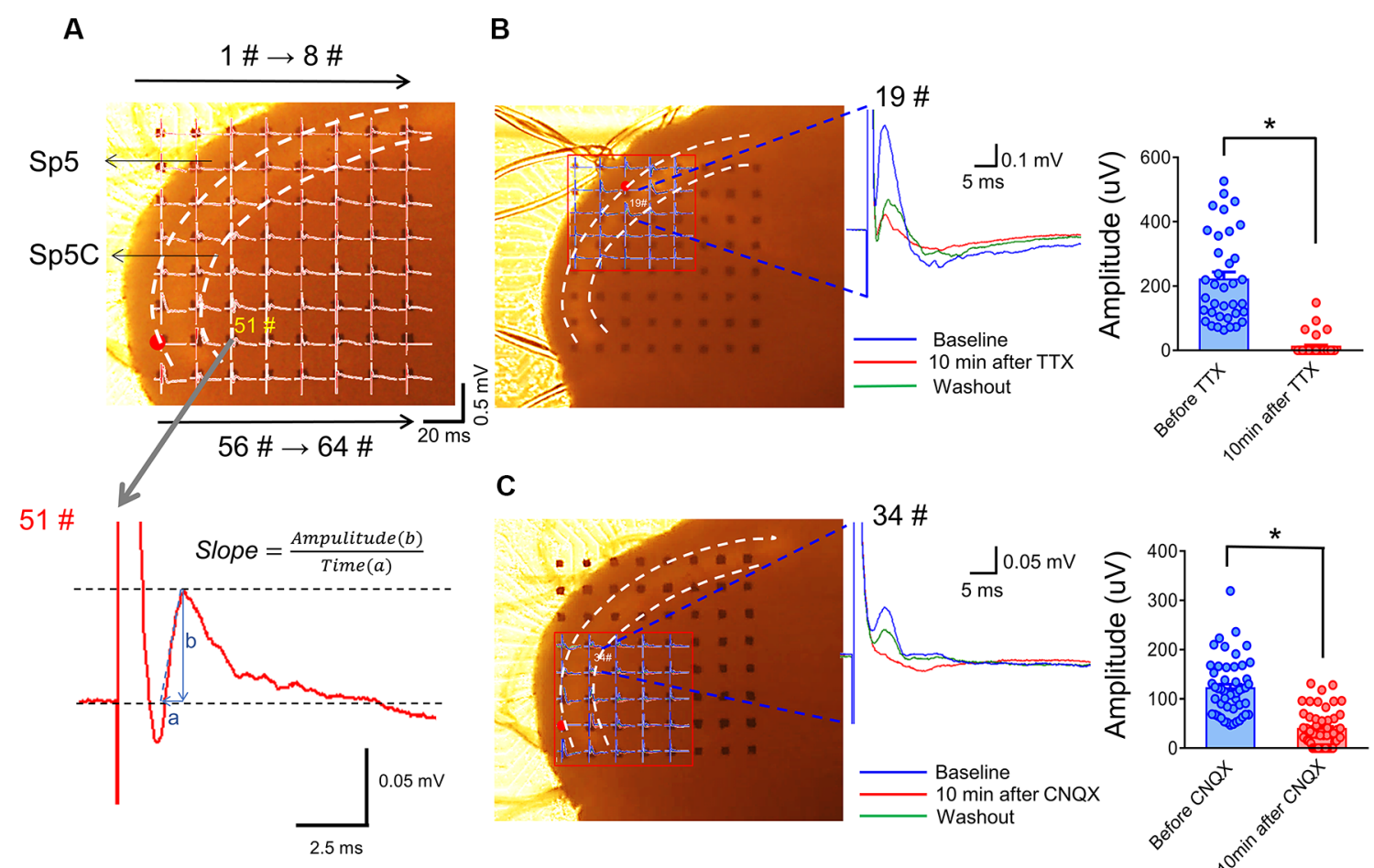

C
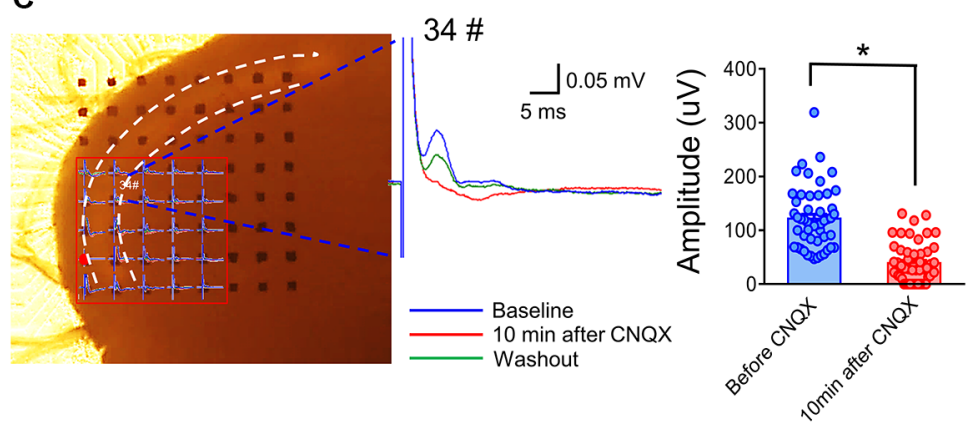

FIGURE 4 | Arrangement of an acutely dissociated medullary dorsal horn slice on an MEA recording dish and pharmacological identification of evoked network local field potentials (eLFPs). Panel (A) shows a typical example of the MEA (8 $\times$ 8, 64 electrodes) recordings on a rat medullary dorsal horn (Sp5C) slice. The upper panel shows representative network traces of eLFPs recorded across the 63 sites following electrical stimulation at the spinal tract of the trigeminal nerve (Sp5, red dot for \#49). The vertical scale indicates the amplitude of potentials ( $0.5 \mathrm{mV}$ ), horizontal scale indicates the time (20 ms). The lower panel showing a typical waveform of eLFPs recorded from 51\# electrode (gray arrow), the slope was calculated as the ratio of amplitude (b) to a time interval (a). Panel (B) shows blocking effect of eLFPs by perfusion with TTX (1 $\mu \mathrm{M}$; dots mean 36 electrode points from $n=6$ slices). Panel (C) shows the suppressive effect of eLFPs by perfusion with CNQX (10 $\mu \mathrm{M}$; dots mean 66 electrode points from $n=8$ slices). ${ }^{*} p<0.05$, drug vs. baseline. Error bars: \pm SEM. 

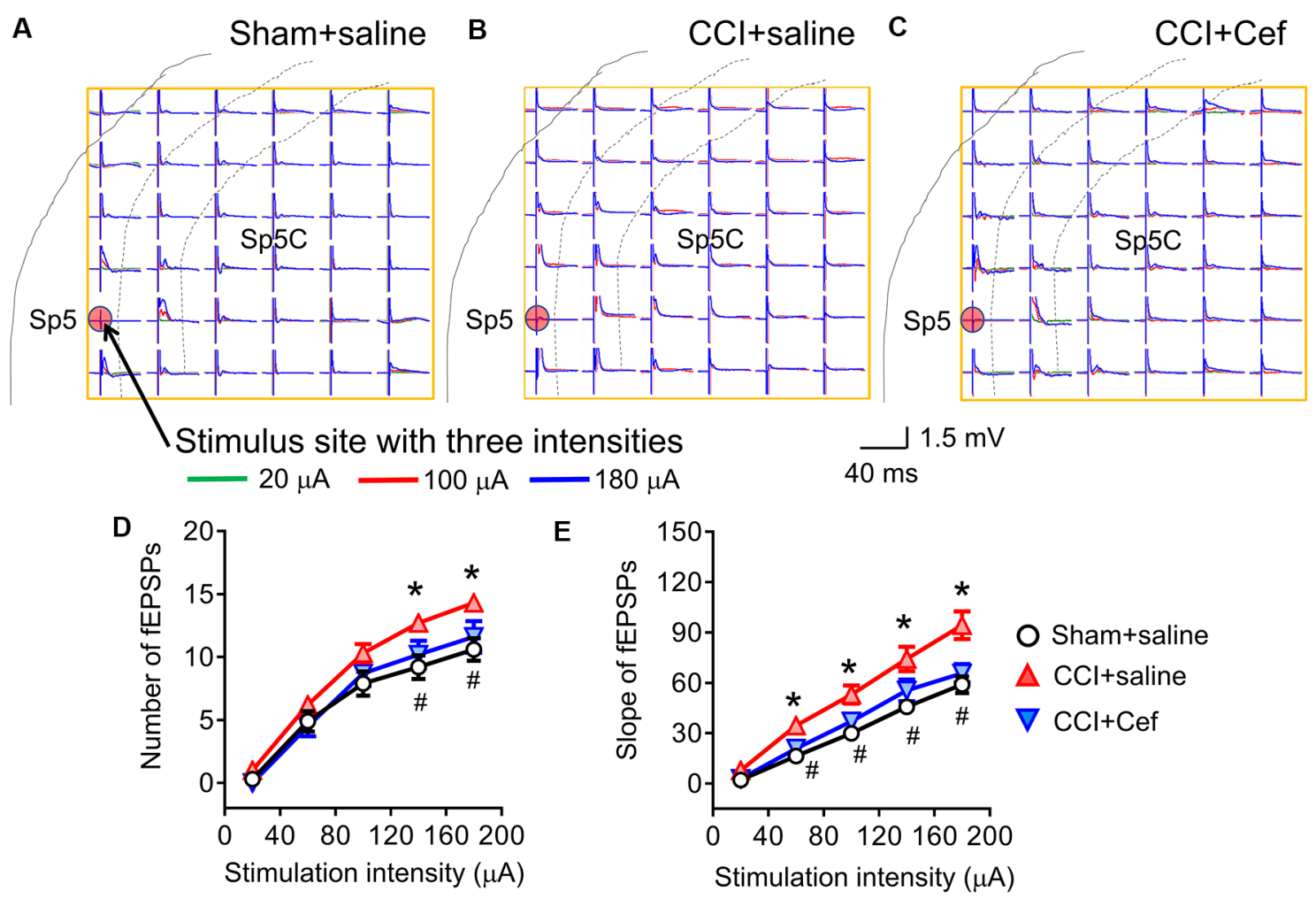

$\longrightarrow 1.5 \mathrm{mV}$

$180 \mu \mathrm{A}$

$40 \mathrm{~ms}$

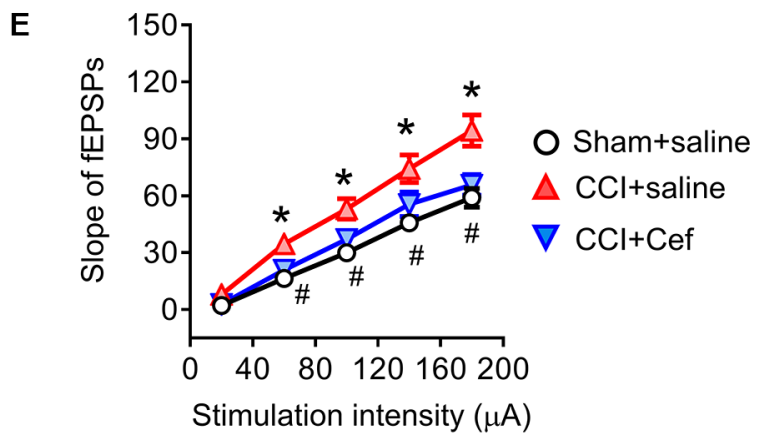

FIGURE 5 | Reversal effects of repeated systemic administration of ceftriaxone (Cef) on CCI-ION-induced enhancement of network field excitatory postsynaptic potentials (fEPSPs) response. Panels (A-C) shows typical examples of the MEA $(8 \times 8,64$ electrodes) recordings on a rat medullary dorsal horn (Sp5C) slice from Sham + saline, $\mathrm{CCl}+$ saline and $\mathrm{CCl}+$ Cef groups following three intensities (overlapping traces: green for $20 \mu \mathrm{A}$, red for $100 \mu \mathrm{A}$ and blue for $180 \mu \mathrm{A}$ ) of electrical stimulation at the Sp5 (red dot). Panel (D) shows the average number of evoked fEPSPs following electrical stimulation with a series of intensities ranging from $20 \mu \mathrm{A}$ to $199 \mu \mathrm{A}$ in Sham + saline $(n=10), \mathrm{CCl}+$ saline $(n=10)$ and CCl-Cef $(n=9)$ groups. Panel (E) shows averaged slope of evoked fEPSPs following electrical stimulation with a series of intensities ranging from $20 \mu \mathrm{A}$ to $199 \mu \mathrm{A}$ in Sham + saline $(n=10), \mathrm{CCl}+\mathrm{saline}(n=10)$ and CCl-Cef $(n=9)$ groups. The data of panels (D,E) are presented as mean \pm SEM. ${ }^{*} p<0.05, \mathrm{CCl}+$ saline vs. Sham + saline; ${ }^{*} p<0.05, \mathrm{CCl}+\mathrm{Cef} v \mathrm{~s}$. CCl + saline.

effects on the basal pain sensitivity, suggesting a potential therapeutic use of the drug in prevention of clinical-pathological pain; (2) the therapeutic effect of Cef on the CCI-IONinduced TN was through restoration of GLT-1 expression in the medullary dorsal horn that had been deficient during the neuropathic processes; (3) the GLT-1 deficiency could lead to dysfunctional synaptic plasticity in the medullary dorsal horn, displaying as enhancement of both spatial widespread and temporal elongation of network synaptic responses in terms of increased number of fEPSPs, leftward shift of I-V curve, and enhanced LTP induction and maintenance; and (4) repeated systemic administration of Cef could reverse the abnormal spatiotemporal synaptic plasticity through restoration of GLT-1 expression in the medullary dorsal horn under the CCI-IONinduced trigeminal neuropathic condition.

\section{Contribution of Medullary Dorsal Horn GLT-1 Deficiency to the Development of Trigeminal Neuropathic Pain}

Glutamate serves as a major excitatory neurotransmitter in the central nervous system (CNS) and plays a critical role in excitatory synaptic transmission via acting at its ionic
(AMPA/NMDA) and/or metabotropic receptors (Ozawa et al., 1998). It is well known that both peripheral tissue and nerve injuries can cause central sensitization or abnormal synaptic plasticity in the spinal dorsal horn that involves glutamatergic neurotransmission (Woolf and Thompson, 1991; Larsson and Broman, 2011). However, over the past decades, a huge number of studies have been focused on the therapeutic strategy targeting antagonists and blockers of various subtypes of glutamate receptors but with fewer being concentrating on how to restore dysfunctions of glutamate release, reuptake and metabolism during the processes of pathological pain. Long-term abnormal extracellular elevation of spinal glutamate release has been previously found to be caused by peripheral inflammatory pain (Yan et al., 2009). The imbalance between excitatory and inhibitory synaptic transmission and modulation has also been revealed to be existent in various CNS regions due to increased excitatory amino acids (EAAs) and decreased inhibitory amino acids (IAAs) under both neuropathic and inflammatory pain conditions (Yan et al., 2009; Gong et al., 2010; Wang et al., 2018; Cao et al., 2019). Those previous results highly suggest that abnormal reuptake of presynaptic glutamate release by astrocytic GLT-1 or GLAST may lead to synaptic cleft accumulation of 


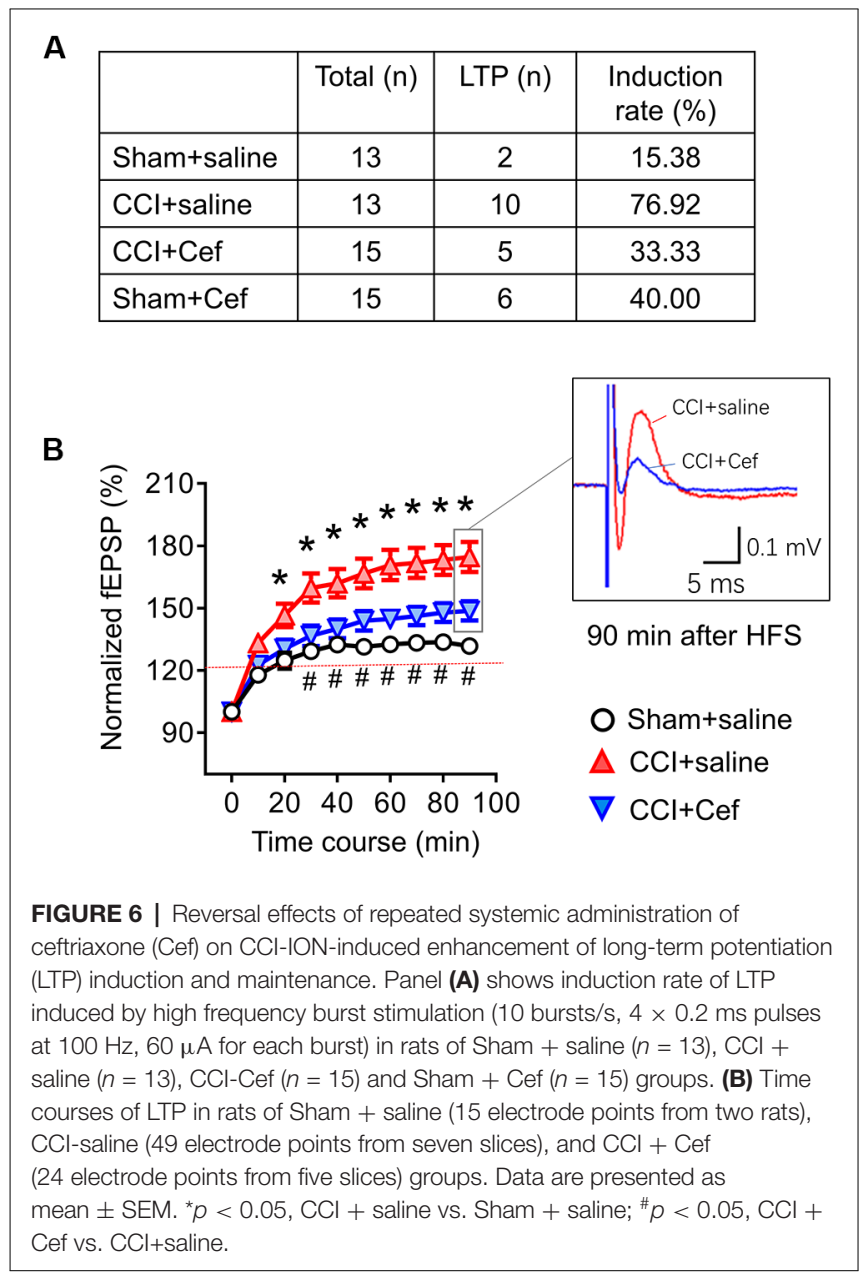

EAAs that persistently activate post-synaptic AMPA/NMDA receptors and downstream intracellular cascades, resulting in enhancement of EPSPs associated with chronic pain (Danbolt, 2001; Kanai et al., 2013). As lines of evidence, astrocytic GLT-1 expression could be impaired by both spinal cord and peripheral nerve injuries, leading to down-regulation of GLT-1 protein and an increase in extracellular glutamate accumulation (Rao et al., 1998; Jacob et al., 2007; Wang et al., 2018). Moreover, abnormal down-regulation of GLT-1 has also been demonstrated to be associated with the development of neuropathic pain induced by CCI-SN (Suzuki et al., 2012), SNL (Hobo et al., 2011) and SNI (Inquimbert et al., 2012). Elsewhere, a transient up-regulation of GLT-1 was observed in some pain models at the initial phase (Yamada et al., 1998; Cavaliere et al., 2007), which was thought to be a compensatory effect in response to the increasing glutamate release, however, the expression of GLT-1 was stably down-regulated at the later phase of the peripheral nerve injury (Weng et al., 2014; Yan et al., 2014), implicating potential contribution of medullary dorsal horn GLT-1 deficiency to development of TNP. In the current study, we provided a new line of evidence supporting this presumption by different levels of assays in terms of behavioral, cellular and molecular, and electrophysiological techniques. Meanwhile, we demonstrated that repeated systemic administration of Cef for at least 5 days could restore protein expression of GLT-1 at the medullary dorsal horn wherein the CCI-ION-induced abnormal spatiotemporal synaptic plasticity was subsequently rescued and pain hypersensitivity was greatly improved. Because the rescue effects of Cef to up-regulate GLT-1 expression and to increase glutamate reuptake have also been demonstrated to be responsible for the relief of neuropathic pain due to other origins (Hu et al., 2010; Ramos et al., 2010; Butler, 2018) and repeated systemic administration of Cef did not affect basal pain sensitivity in normal and sham rats, this class of structural analogs might be promising in the management of neuropathic pain in the clinic. Although repeated administration of Cef at the acute phase (day 1 after the CCI-ION) was demonstrated to be effective in the prevention of the TNP condition from occurring in the current study, it is still unknown whether the same Cef treatment would be effective to relieve chronic phase of the TNP. Thus, a future study is required to be carried out to look at the therapeutic analgesic effect of Cef under the chronic phase of the TNP.

\section{Contribution of Medullary Dorsal Horn GLT-1 Deficiency to Abnormal Spatiotemporal Synaptic Plasticity Induced by Pathological Pain}

It is well known that both tissue and nerve injury in the periphery can cause spatiotemporal synaptic plasticity in the CNS of different levels from spinal dorsal horn to the primary somatosensory cortex (S1), anterior cingulate cortex (ACC) and hippocampus (Gong et al., 2010; Lyu et al., 2013; Lu et al., 2014; Yu et al., 2017; Wang et al., 2018; Cao et al., 2019). The spatiotemporal synaptic plasticity when abnormally lasts can be associated with development and maintenance of chronic pain and its comorbidities including emotional disorders (anxiety and depression) and cognitive deficits (Gong et al., 2010; Lyu et al., 2013; Liu and Chen, 2014; Lu et al., 2014; Yu et al., 2017; Wang et al., 2018; Cao et al., 2019). At the single synaptic level, slice whole-cell recordings have demonstrated that the frequency of miniature and spontaneous excitatory postsynaptic currents (mEPSCs/sEPSCs) could be greatly increased, while that of inhibitory postsynaptic currents (mIPSCs/sIPSCs) could be distinctly decreased under pathological pain condition, implicating an imbalance between EAAs and IAAs release from pre-synaptic component (Gong et al., 2010; Wang et al., 2018; Cao et al., 2019). This imbalance between EAAs and IAAs releases at the synaptic cleft is likely to be responsible for abnormal spatiotemporal synaptic plasticity displaying as the enhanced amplitude of fEPSPs, the leftward shift of I-V curve and increased LTP induction rate and magnitude (Zhao et al., 2009; Gong et al., 2010; Lyu et al., 2013; Lu et al., 2014; Wang et al., 2018; Cao et al., 2019). Moreover, the pathological pain associated imbalance between EAAs and IAAs releases at the synaptic cleft was able to cause translocation or trafficking of GluR1 from cytosol to cell membrane (i.e., externalization), while GluR2 and $\mathrm{GABA}_{\mathrm{A}} 1 \alpha$ from the membrane to the cytosol (i.e., internalization), facilitating induction and magnification of LTP in association with NMDA receptors (Cao et al., 2019). In the current study, we further demonstrated that the 
CCI-ION could produce spatiotemporal synaptic plasticity at the medullary dorsal horn as well displaying an enhanced amplitude of fEPSPs, the leftward shift of I-V curve and increased LTP induction rate and magnitude. The CCI-ION-induced spatiotemporal synaptic plasticity could be fully reversed by repeated systemic administration of Cef which could restore the GLT-1 expression, suggesting a contribution of medullary dorsal horn GLT-1 deficiency to abnormal spatiotemporal synaptic plasticity induced by neuropathic pain. Namely, the CCI-IONinduced medullary dorsal horn GLT-1 deficiency would lead to the accumulation of glutamate at the synaptic cleft, resulting in persistent activation of ionic AMPA/NMDA receptors and intracellular cascades that are required for LTP induction and maintenance. This process may be exaggerated by magnified primary afferents (Sp5) maintained by excessive extracellular glutamate accumulation-triggered intracellular $\mathrm{Ca}^{2+}$ release in astrocytic cells (Rojas et al., 2007; Yoshizumi et al., 2012). It is well known that the rate of diffusion is one of the major parameters in glutamate clearance (Piet et al., 2004), and this process is accelerated by glutamate transporters (e.g., GLT-1) expressed in astrocytic cells (Tzingounis and Wadiche, 2007). The abnormal deficiency of glutamate transporters could directly influence the clearance of glutamate, subsequently leading to glutamate spills over the synaptic cleft and diffuses to distant synapses. At the spatial network level, because previous studies have shown that widespread activation of distant neurons and astrocytic cells could be elicited by widespread extracellular glutamate accumulation due to the GLT-1 deficiency in the spinal dorsal horn (Nie and Weng, 2010; Nie et al., 2010), network widespread expansion of fEPSPs observed in our current and previous studies (Zhao et al., 2009; Lyu et al., 2013; Lu et al., 2014; Cao et al., 2019) could be well explained. Of course, activation of glutamate mGluRs, the release of $\mathrm{GABA}_{\mathrm{A}}$-mediated tonic inhibition and activation of silent synapses could also be involved in this processing (Voronin and Cherubini, 2004).

As for the reversal effect of Cef on the CCI-ION-induced GLT-1 deficiency, little is known about its mechanistic actions. In the current study, it was revealed that repeated systemic administration of Cef could up-regulate the expression of GLT-1 in the medullary dorsal horn, suggesting its direct or indirect genetic or epigenetic modulation of GLT-1 genes in certain astrocytic cells. Some previous studies have found the phenomenon that Cef could relieve neuropathic pain through up-regulation of GLT-1, but it could not fully explain how Cef works at the molecular and cellular levels by some presumptions, i.e., astrocytic inactivation (Nicholson et al., 2014) or astrocytic-neuronal bidirectional communications (Pasti et al.,

\section{REFERENCES}

Bonansco, C., and Fuenzalida, M. (2016). Plasticity of hippocampal excitatoryinhibitory balance: missing the synaptic control in the epileptic brain. Neural. Plast. 2016:8607038. doi: 10.1155/2016/8607038

Butler, S. (2018). Effects of the excitatory amino acid transporter subtype 2 (EAAT2) transporter inducer ceftriaxone (an antibiotic) on different pain modalities in rat. Scand. J. Pain 2, 130-131. doi: 10.1016/j.sjpain.2011.05.003

Cao, F. L., Xu, M., Gong, K. R., Wang, Y., Wang, R. R., Chen, X. F., et al. (2019). Imbalance between excitatory and inhibitory synaptic transmission in
1997; Ni et al., 2007). Theoretically, direct or indirect genetic or epigenetic modulation of GLT-1 genes in certain astrocytic cells by Cef could be explainable for the 10-days delayed analgesic effects following 5-days systemic administration of Cef observed in the current study. The underlying mechanisms of Cef-induced restoration of GLT-1 expression remain unclear and require to be further studied in the future.

In summary: Cef can relieve TNP through suppression of spatiotemporal synaptic plasticity via GLT-1 restoration in the medullary dorsal horn of the trigeminal nerve and is promising as a novel therapeutic target for the treatment of $\mathrm{TN}$, in particular, and other neuropathic pains, in general.

\section{DATA AVAILABILITY STATEMENT}

All datasets presented in this study are included in the article/ Supplementary Material.

\section{ETHICS STATEMENT}

The animal study was reviewed and approved by Institutional Animal Care and Use Committee at the Fouth Military Medical University.

\section{AUTHOR CONTRIBUTIONS}

$\mathrm{DH}$ and JC designed and managed the research work. XL, TH, J-LW, R-RW, and X-LW performed the experiments and collected the data. XL, TH, YW, X-BY, H-CZ, RD, and JC contributed to the analyses and plotting of the data. JC, XL, and $\mathrm{DH}$ composed the manuscript.

\section{FUNDING}

This work was supported partially by grants from National Natural Science Foundation of China to DH (81771101), JC (81571072 and 31771159) and YW (31500854). This work was also supported partially by the Hunan Provincial Natural Science Foundation of China (Grant No. 2019JJ40462) and Key lab of Hunan Province grant (No. 2018TP1009).

\section{SUPPLEMENTARY MATERIAL}

The Supplementary Material for this article can be found online at: https://www.frontiersin.org/articles/10.3389/fncel. 2020.00199/full\#supplementary-material.

the primary somatosensory cortex caused by persistent nociception in rats. J. Pain 20, 917-931. doi: 10.1016/j.jpain.2018.11.014

Cavaliere, C., Cirillo, G., Rosaria Bianco, M., Rossi, F., De Novellis, V., Maione, S., et al. (2007). Gliosis alters expression and uptake of spinal glial amino acid transporters in a mouse neuropathic pain model. Neuron. Glia Biol. 3, 141-153. doi: 10.1017/s1740925x07000695

Chelini, A., Brogi, S., Paolino, M., Di Capua, A., Cappelli, A., Giorgi, G., et al. (2017). Synthesis and biological evaluation of novel neuroprotective pyridazine derivatives as excitatory amino acid transporter 2 (EAAT2) activators. J. Med. Chem. 60, 5216-5221. doi: 10.1021/acs.jmedchem.7b00383 
Chen, W., Aoki, C., Mahadomrongkul, V., Gruber, C. E., Wang, G. J., Blitzblau, R., et al. (2002). Expression of a variant form of the glutamate transporter GLT1 in neuronal cultures and in neurons and astrocytes in the rat brain. J. Neurosci. 22, 2142-2152. doi: 10.1523/jneurosci.22-06-02142.2002

Chen, W., Mahadomrongkul, V., Berger, U. V., Bassan, M., DeSilva, T., Tanaka, K., et al. (2004). The glutamate transporter GLTla is expressed in excitatory axon terminals of mature hippocampal neurons. J. Neurosci. 24, 1136-1148. doi: 10.1523/jneurosci.1586-03.2004

Cheng, J. S., Lim, D. A., Chang, E. F., and Barbaro, N. M. (2014). A review of percutaneous treatments for trigeminal neuralgia. Neurosurgery 10, 25-33; discussion 33. doi: 10.1227/NEU.00000000000001687

Danbolt, N. C. (2001). Glutamate uptake. Prog. Neurobiol. 65, 1-105. doi: 10.1016/s0301-0082(00)00067-8

Di Cesare Mannelli, L., Marcoli, M., Micheli, L., Zanardelli, M., Maura, G., Ghelardini, C., et al. (2015). Oxaliplatin evokes P2X7-dependent glutamate release in the cerebral cortex: a pain mechanism mediated by Pannexin 1 . Neuropharmacology 97, 133-141. doi: 10.1016/j.neuropharm.2015.05.037

Finnerup, N. B., Sindrup, S. H., and Jensen, T. S. (2010). The evidence for pharmacological treatment of neuropathic pain. Pain 150, 573-581. doi: 10.1016/j.pain.2010.06.019

Furness, D. N., Dehnes, Y., Akhtar, A. Q., Rossi, D. J., Hamann, M., Grutle, N. J., et al. (2008). A quantitative assessment of glutamate uptake into hippocampal synaptic terminals and astrocytes: new insights into a neuronal role for excitatory amino acid transporter 2 (EAAT2). Neuroscience 157, 80-94. doi: 10.1016/j.neuroscience.2008.08.043

Gong, K. R., Cao, F. L., He, Y., Gao, C. Y., Wang, D. D., Li, H., et al. (2010). Enhanced excitatory and reduced inhibitory synaptic transmission contribute to persistent pain-induced neuronal hyper-responsiveness in anterior cingulate cortex. Neuroscience 171, 1314-1325. doi: 10.1016/j.neuroscience.2010.10.028

Gunduz, O., Oltulu, C., Buldum, D., Guven, R., and Ulugol, A. (2011). Antiallodynic and anti-hyperalgesic effects of ceftriaxone in streptozocin-induced diabetic rats. Neurosci. Lett. 491, 23-25. doi: 10.1016/j.neulet.2010.12.063

Hazell, A. S., Sheedy, D., Oanea, R., Aghourian, M., Sun, S., Jung, J. Y., et al. (2010). Loss of astrocytic glutamate transporters in Wernicke encephalopathy. Glia 58, 148-156. doi: $10.1002 /$ glia.20908

Heusler, P., Cebulla, B., Boehmer, G., and Dinse, H. R. (2000). A repetitive intracortical microstimulation pattern induces long-lasting synaptic depression in brain slices of the rat primary somatosensory cortex. Exp. Brain. Res. 135, 300-310. doi: 10.1007/s002210000530

Hjornevik, T., Jacobsen, L. M., Qu, H., Bjaalie, J. G., Gjerstad, J., and Willoch, F. (2008). Metabolic plasticity in the supraspinal pain modulating circuitry after noxious stimulus-induced spinal cord LTP. Pain 140, 456-464. doi: 10.1016/j. pain.2008.09.029

Hobo, S., Eisenach, J. C., and Hayashida, K. (2011). Up-regulation of spinal glutamate transporters contributes to anti-hypersensitive effects of valproate in rats after peripheral nerve injury. Neurosci. Lett. 502, 52-55. doi: 10.1016/j. neulet.2011.07.023

Hu, Y., Li, W., Lu, L., Cai, J., Xian, X., Zhang, M., et al. (2010). An anti-nociceptive role for ceftriaxone in chronic neuropathic pain in rats. Pain 148, 284-301. doi: 10.1016/j.pain.2009.11.014

Imamura, Y., Kawamoto, H., and Nakanishi, O. (1997). Characterization of heat-hyperalgesia in an experimental trigeminal neuropathy in rats. Exp. Brain. Res. 116, 97-103. doi: 10.1007/pl00005748

Inquimbert, P., Bartels, K., Babaniyi, O. B., Barrett, L. B., Tegeder, I., and Scholz, J. (2012). Peripheral nerve injury produces a sustained shift in the balance between glutamate release and uptake in the dorsal horn of the spinal cord. Pain 153, 2422-2431. doi: 10.1016/j.pain.2012.08.011

Jacob, C. P., Koutsilieri, E., Bartl, J., Neuen-Jacob, E., Arzberger, T., Zander, N., et al. (2007). Alterations in expression of glutamatergic transporters and receptors in sporadic Alzheimer's disease. J. Alzheimers Dis. 11, 97-116. doi: $10.3233 /$ jad-2007-11113

Kanai, Y., Clemencon, B., Simonin, A., Leuenberger, M., Lochner, M., Weisstanner, M., et al. (2013). The SLC1 high-affinity glutamate and neutral amino acid transporter family. Mol. Aspects. Med. 34, 108-120. doi: 10.1016/j. mam.2013.01.001

Katagiri, H., Tanaka, K., and Manabe, T. (2001). Requirement of appropriate glutamate concentrations in the synaptic cleft for hippocampal LTP induction. Eur. J. Neurosci. 14, 547-553. doi: 10.1046/j.0953-816x.2001.01664.x
Larsson, M., and Broman, J. (2011). Synaptic plasticity and pain: role of ionotropic glutamate receptors. Neuroscientist 17, 256-273. doi: $10.1177 / 1073858409349913$

Lepore, A. C., O’Donnell, J., Bonner, J. F., Paul, C., Miller, M. E., Rauck, B., et al. (2011). Spatial and temporal changes in promoter activity of the astrocyte glutamate transporter GLT1 following traumatic spinal cord injury. J. Neurosci. Res. 89, 1001-1017. doi: 10.1002/jnr.22624

Levenson, J., Weeber, E., Selcher, J. C., Kategaya, L. S., Sweatt, J. D., and Eskin, A. (2002). Long-term potentiation and contextual fear conditioning increase neuronal glutamate uptake. Nat. Neurosci. 5, 155-161. doi: 10.1038/nn791

Liu, M. G., and Chen, J. (2014). Preclinical research on pain comorbidity with affective disorders and cognitive deficits: challenges and perspectives. Prog. Neurobiol. 116, 13-32. doi: 10.1016/j.pneurobio.2014.01.003

Liu, X. J., and Salter, M. W. (2010). Glutamate receptor phosphorylation and trafficking in pain plasticity in spinal cord dorsal horn. Eur. J. Neurosci. 32, 278-289. doi: 10.1111/j.1460-9568.2010.07351.x

Lu, Y. F., Wang, Y., He, Y., Zhang, F. K., He, T., Wang, R. R., et al. (2014). Spatial and temporal plasticity of synaptic organization in anterior cingulate cortex following peripheral inflammatory pain: multi-electrode array recordings in rats. Neurosci. Bull. 30, 1-20. doi: 10.1007/s12264-013-1344-9

Lyu, D., Yu, W., Tang, N., Wang, R., Zhao, Z., Xie, F., et al. (2013). The mTOR signaling pathway regulates pain-related synaptic plasticity in rat entorhinalhippocampal pathways. Mol. Pain 9:64. doi: 10.1186/1744-8069-9-64

Malinow, R., and Malenka, R. C. (2002). AMPA receptor trafficking and synaptic plasticity. Annu. Rev. Neurosci. 25, 103-126. doi: 10.1146/annurev.neuro.25. 112701.142758

Ni, Y., Malarkey, E. B., and Parpura, V. (2007). Vesicular release of glutamate mediates bidirectional signaling between astrocytes and neurons. J. Neurochem. 103, 1273-1284. doi: 10.1111/j.1471-4159.2007.04864.x

Nicholson, K. J., Gilliland, T. M., and Winkelstein, B. A. (2014). Upregulation of GLT-1 by treatment with ceftriaxone alleviates radicular pain by reducing spinal astrocyte activation and neuronal hyperexcitability. J. Neurosci. Res. 92, 116-129. doi: 10.1002/jnr.23295

Nie, H., and Weng, H. R. (2010). Impaired glial glutamate uptake induces extrasynaptic glutamate spillover in the spinal sensory synapses of neuropathic rats. J. Neurophysiol. 103, 2570-2580. doi: 10.1152/jn.00013.2010

Nie, H., Zhang, H., and Weng, H. R. (2010). Bidirectional neuron-glia interactions triggered by deficiency of glutamate uptake at spinal sensory synapses. J. Neurophysiol. 104, 713-725. doi: 10.1152/jn.00282.2010

Ozawa, S., Kamiya, H., and Tsuzuki, K. (1998). Glutamate receptors in the mammalian central nervous system. Prog. Neurobiol. 54, 581-618. doi: 10.1016/s0301-0082(97)00085-3

Pasti, L., Volterra, A., Pozzan, T., and Carmignoto, G. (1997). Intracellular calcium oscillations in astrocytes: a highly plastic, bidirectional form of communication between neurons and astrocytes in situ. J. Neurosci. 17, 7817-7830. doi: 10.1523/jneurosci.17-20-07817.1997

Piet, R., Vargova, L., Sykova, E., Poulain, D. A., and Oliet, S. H. (2004). Physiological contribution of the astrocytic environment of neurons to intersynaptic crosstalk. Proc. Natl. Acad. Sci. U S A 101, 2151-2155. doi: 10.1073/pnas.0308408100

Ramos, K. M., Lewis, M. T., Morgan, K. N., Crysdale, N. Y., Kroll, J. L., Taylor, F. R., et al. (2010). Spinal upregulation of glutamate transporter GLT-1 by ceftriaxone: therapeutic efficacy in a range of experimental nervous system disorders. Neuroscience 169, 1888-1900. doi: 10.1016/j.neuroscience.2010. 06.014

Rao, V. L., Baskaya, M. K., Dogan, A., Rothstein, J. D., and Dempsey, R. J. (1998). Traumatic brain injury down-regulates glial glutamate transporter (GLT-1 and GLAST) proteins in rat brain. J. Neurochem. 70, 2020-2027. doi: 10.1046/j. 1471-4159.1998.70052020.x

Rawls, S. M., Zielinski, M., Patel, H., Sacavage, S., Baron, D. A., and Patel, D. (2010). Beta-lactam antibiotic reduces morphine analgesic tolerance in rats through GLT-1 transporter activation. Drug Alcohol. Depend. 107, 261-263. doi: 10.1016/j.drugalcdep.2009.10.010

Rojas, H., Colina, C., Ramos, M., Benaim, G., Jaffe, E. H., Caputo, C., et al. (2007). $\mathrm{Na}^{+}$entry via glutamate transporter activates the reverse $\mathrm{Na}^{+} / \mathrm{Ca}^{2+}$ exchange and triggers $\mathrm{Ca}_{\mathrm{i}}^{2+}$-induced $\mathrm{Ca}^{2+}$ release in rat cerebellar Type-1 astrocytes. J. Neurochem. 100, 1188-1202. doi: 10.1111/j.1471-4159.2006. 04303.x 
Rose, E. M., Koo, J. C., Antflick, J. E., Ahmed, S. M., Angers, S., and Hampson, D. R. (2009). Glutamate transporter coupling to Na,K-ATPase. J. Neurosci. 29, 8143-8155. doi: 10.1523/JNEUROSCI.1081-09.2009

Rothstein, J. D., Martin, L., Levey, A. I., Dykes-Hoberg, M., Jin, L., Wu, D., et al. (1994). Localization of neuronal and glial glutamate transporters. Neuron 13, 713-725. doi: 10.1016/0896-6273(94)90038-8

Rothstein, J. D., Patel, S., Regan, M. R., Haenggeli, C., Huang, Y. H., Bergles, D. E., et al. (2005). Beta-lactam antibiotics offer neuroprotection by increasing glutamate transporter expression. Nature 433, 73-77. doi: 10.1038/nature03180

Rothstein, J. D., Van Kammen, M., Levey, A. I., Martin, L. J., and Kuncl, R. W. (1995). Selective loss of glial glutamate transporter GLT-1 in amyotrophic lateral sclerosis. Ann. Neurol. 38, 73-84. doi: 10.1002/ana.410380114

Scholz, J., Finnerup, N. B., Attal, N., Aziz, Q., Baron, R., Bennett, M. I., et al. (2019). The IASP classification of chronic pain for ICD-11: chronic neuropathic pain. Pain 160, 53-59. doi: 10.1097/j.pain.0000000000001365

Suzuki, Y., Mitsuhata, H., Yuzurihara, M., and Kase, Y. (2012). Antiallodynic effect of herbal medicine yokukansan on peripheral neuropathy in rats with chronic constriction injury. Evid. Based. Complement. Alternat. Med. 2012:953459. doi: 10.1155/2012/953459

Tang, F. R., and Lee, W. L. (2001). Expression of the group II and III metabotropic glutamate receptors in the hippocampus of patients with mesial temporal lobe epilepsy. J. Neurocytol. 30, 137-143. doi: 10.1023/a:1011939223872

Ting, J. T., Daigle, T. L., Chen, Q., and Feng, G. (2014). Acute brain slice methods for adult and aging animals: application of targeted patch clamp analysis and optogenetics. Methods Mol. Biol. 1183, 221-242. doi: 10.1007/978-1-49391096-0_14

Tronnier, V. M., Rasche, D., Hamer, J., Kienle, A. L., and Kunze, S. (2001). Treatment of idiopathic trigeminal neuralgia: comparison of long-term outcome after radiofrequency rhizotomy and microvascular decompression. Neurosurgery 48, 1261-1267; discussion 1267-1268. doi: 10.1097/00006123200106000-00014

Tzingounis, A. V., and Wadiche, J. I. (2007). Glutamate transporters: confining runaway excitation by shaping synaptic transmission. Nat. Rev. Neurosci. 8, 935-947. doi: 10.1038/nrn2274

Voronin, L. L., and Cherubini, E. (2004). 'Deaf, mute and whispering' silent synapses: their role in synaptic plasticity. J. Physiol. 557, 3-12. doi: 10.1113/jphysiol.2003.058966

Vos, B. P., Strassman, A. M., and Maciewicz, R. J. (1994). Behavioral evidence of trigeminal neuropathic pain following chronic constriction injury to the rat's infraorbital nerve. J. Neurosci. 14, 2708-2723. doi: 10.1523/jneurosci.14-0502708.1994

Wang, D. D., Li, Z., Chang, Y., Wang, R. R., Chen, X. F., Zhao, Z. Y., et al. (2010). Neural circuits and temporal plasticity in hindlimb representation of rat primary somatosensory cortex: revisited by multi-electrode array on brain slices. Neurosci. Bull. 26, 175-187. doi: 10.1007/s12264-010-0308-6

Wang, R. R., Wang, Y., Guan, S. M., Li, Z., Kokane, S., Cao, F. L., et al. (2018). Synaptic homeostasis and allostasis in the dentate gyrus caused by inflammatory and neuropathic pain conditions. Front. Synaptic. Neurosci. 10:1 doi: 10.3389/fnsyn.2018.00001

Weng, H. R., Gao, M., and Maixner, D. W. (2014). Glycogen synthase kinase 3 beta regulates glial glutamate transporter protein expression in the spinal dorsal horn in rats with neuropathic pain. Exp. Neurol. 252, 18-27. doi: 10.1016/j. expneurol.2013.11.018

Woolf, C. J., and Thompson, S. W. (1991). The induction and maintenance of central sensitization is dependent on N-methyl-D-aspartic acid receptor activation; implications for the treatment of post-injury pain hypersensitivity states. Pain 44, 293-299. doi: 10.1016/0304-3959(91) 90100-c

Yamada, K., Watanabe, M., Shibata, T., Nagashima, M., Tanaka, K., and Inoue, Y. (1998). Glutamate transporter GLT-1 is transiently localized on growing axons of the mouse spinal cord before establishing astrocytic expression. J. Neurosci. 18, 5706-5713. doi: 10.1523/jneurosci.18-15-05706.1998

Yan, L. H., Hou, J. F., Liu, M. G., Li, M. M., Cui, X. Y., Lu, Z. M., et al. (2009). Imbalance between excitatory and inhibitory amino acids at spinal level is associated with maintenance of persistent pain-related behaviors. Pharmacol. Res. 59, 290-299. doi: 10.1016/j.phrs.2009.01.012

Yan, X., Yadav, R., Gao, M., and Weng, H. R. (2014). Interleukin-1 beta enhances endocytosis of glial glutamate transporters in the spinal dorsal horn through activating protein kinase C. Glia 62, 1093-1109. doi: 10.1002/glia. 22665

Yoshizumi, M., Eisenach, J. C., and Hayashida, K. (2012). Riluzole and gabapentinoids activate glutamate transporters to facilitate glutamate-induced glutamate release from cultured astrocytes. Eur. J. Pharmacol. 677, 87-92. doi: 10.1016/j.ejphar.2011.12.015

Yu, F., Zhao, Z. Y., He, T., Yu, Y. Q., Li, Z., and Chen, J. (2017). Temporal and spatial dynamics of peripheral afferent-evoked activity in the dorsal horn recorded in rat spinal cord slices. Brain Res. Bull. 131, 183-191. doi: 10.1016/j. brainresbull.2017.04.012

Zhao, X. Y., Liu, M. G., Yuan, D. L., Wang, Y., He, Y., Wang, D. D., et al. (2009). Nociception-induced spatial and temporal plasticity of synaptic connection and function in the hippocampal formation of rats: a multi-electrode array recording. Mol. Pain 5:55. doi: 10.1186/1744-8069-5-55

Conflict of Interest: The authors declare that the research was conducted in the absence of any commercial or financial relationships that could be construed as a potential conflict of interest.

Copyright (c) 2020 Luo, He, Wang, Wang, Yan, Zhou, Wang, Du, Wang, Chen and Huang. This is an open-access article distributed under the terms of the Creative Commons Attribution License (CC BY). The use, distribution or reproduction in other forums is permitted, provided the original author(s) and the copyright owner(s) are credited and that the original publication in this journal is cited, in accordance with accepted academic practice. No use, distribution or reproduction is permitted which does not comply with these terms. 